\title{
C1q/TNF-related protein 3 (CTRP3) and 9 (CTRP9) concentrations are decreased in patients with heart failure and are associated with increased morbidity and mortality
}

Chao Gao ${ }^{1+}$, Shasha Zhao ${ }^{1+}$, Kun Lian ${ }^{1}$, Baibing Mi ${ }^{2}$, Rui $\mathrm{Si}^{1}$, Zhijun $\mathrm{Tan}^{3}$, Feng Fu ${ }^{4}$, Shuai Wang ${ }^{1}$, Rutao Wang ${ }^{1}$, Xinliang $\mathrm{Ma}^{5}$ and Ling $\mathrm{TaO}^{1{ }^{*}}$ (D)

\begin{abstract}
Background: Biochemical marker has revolutionized the approach to the diagnosis of heart failure. However, it remains difficult to assess stability of the patient. As such, novel means of stratifying disease severity are needed. C1q/TNF-Related Protein 3 (CTRP3) and C1q/TNF-Related Protein 9 (CTRP9) are novel adipokines that contribute to energy homeostasis with additional anti-inflammatory and anti-ischemic properties. The aim of our study is to evaluate concentrations of CTRP3 and CTRP9 in patients with HFrEF (heart failure with reduced ejection fraction) and whether associated with mortality.

Methods: Clinical data and plasma were obtained from 176 healthy controls and 168 patients with HFrEF. CTRP3 and CTRP9 levels were evaluated by enzyme-linked immunosorbent assay.

Results: Both CTRP3 and CTRP9 concentrations were significantly decreased in the HFrEF group compared to the control group $(p<0.001)$. Moreover, patients with higher New York Heart Association class had significantly lower CTRP3 or CTRP9 concentrations. Correlation analysis revealed that CTRP3 and CTRP9 levels were positively related with LVEF\% (CTRP3, $r=0.556, p<0.001 ;$ CTRP9, $r=0.526, p<0.001)$ and negatively related with NT-proBNP levels (CTRP3, $r=-0.454, p<0.001$; CTRP9, $r=-0.483, p<0.001$ ). After a follow up for 36 months, after adjusted for age, LVEF and NT-proBNP, we observed that CTRP3 or CTRP9 levels below the 25th percentile was a predictor of total mortality (CTRP3,HR:1.93,95\%Cl1.03 3.62,P = 0.042;CTRP9,HR:1.98,95\%Cl:1.02 3.85,P=0.044) and hospitalizations (CTRP3,HR:2.34,95\% Cl:1.43 3.82,P=0.001;CTRP9,HR:2.67,95\%Cl:1.58 4.50,P<0.001).
\end{abstract}

Conclusions: CTRP3 and CTRP9 are decreased in patients with HFrEF, proportionate to disease severity, and each is associated with increased morbidity and mortality.

Trial registration: NCT01372800. Registered May 2011.

Keywords: Biomarker, C1q/TNF-related Protein3 (CTRP3), C1q/TNF-related Protein9 (CTRP9), Heart failure with reduced ejection fraction

\footnotetext{
* Correspondence: lingtao@fmmu.edu.cn

${ }^{+}$Chao Gao and Shasha Zhao contributed equally to this work.

${ }^{1}$ Department of Cardiology, Xijing Hospital, The Fourth Military Medical

University, 15 Changle West Road, Xi'an 710032, China

Full list of author information is available at the end of the article
}

(c) The Author(s). 2019 Open Access This article is distributed under the terms of the Creative Commons Attribution 4.0 International License (http://creativecommons.org/licenses/by/4.0/), which permits unrestricted use, distribution, and reproduction in any medium, provided you give appropriate credit to the original author(s) and the source, provide a link to the Creative Commons license, and indicate if changes were made. The Creative Commons Public Domain Dedication waiver (http://creativecommons.org/publicdomain/zero/1.0/) applies to the data made available in this article, unless otherwise stated. 


\section{Background}

The discovery of novel risk markers for heart failure (HF) has contributed to improved screening, prevention, diagnosis and treatment of HF $[1,2]$. Up to now, BNP and NTproBNP are the most widely used biomarkers in clinical practice, such testing is recommended in current guidelines in determining the diagnosis and prognosis of HF. However, in certain cases, such as for extremely high BNP or NT-proBNP levels, these biomarkers cannot reflect the severity of HF or morphologic changes of the heart. Besides, increased NT-proBNP levels may reflect diminished renal function, left ventricular wall stress, or both. As such, novel means of stratifying disease severity are still needed.

Fat tissue contributes to energy homeostasis and can secrete a number of adipokines that have cardioprotective properties [3]. During heart failure, the secretion of adipokines is dysregulated [4]. However, the dysfunction of adipokines secretion in fat tissue cannot currently be easily measured. Researches have shown adiponectin level was a predictor of mortality, independent of risk markers of $\mathrm{CHF}$ severity. However, the levels of adiponectin in healthy people can range from 5 to $30 \mu \mathrm{g} / \mathrm{ml}$ [5]. It is hard to set the cutoff point between healthy and HF subjects. Interestingly, adiponectin have been recently found belonging to CTRP family [6]. The number of proteins in the CTRP family currently comprises 15 members in addition to adiponectin. Among them, in our previous in vivo studies, we found CTRP (C1q/TNF-Related Protein) 3 and CTRP9 were closely related to cardiovascular diseases [7-9].

CTRP3 is mainly expressed in mammalian subcutaneous and visceral adipocytes [10]. CTRP3 can lower blood glucose in both normal and $o b / o b$ mice without affecting insulin or adiponectin levels [11]. CTRP3 is also found to be an anti-inflammatory adipokine [12] that inhibits proinflammatory pathways such as fatty acid-, LPS- and Toll-like receptor (TLR)-mediated inflammation in monocytes and adipocytes [13]. Moreover, CTRP3 promotes vascular smooth muscle cell proliferation in blood vessel wall after vascular injury [14]. CTRP9 is predominantly expressed in primary adipocytes and stromal cells [15]. CTRP9 can also reduce blood glucose and insulin levels without effecting body weight or food intake. CTRP9 is also a vasorelaxative adipocytokine that may exert vasculoprotective effects via the adiponectin receptor 1/AMPK/eNOS dependent/NO mediated signaling pathway [16]. In an in vivo MI model, we and others found CTRP3 [7] or CTRP9 [17] can improve survival rate, restored cardiac function, attenuated cardiomyocyte apoptosis, attenuated adverse remodeling, increased re-vascularization. However, to date, the levels of CTRP3 and CTRP9 have not been reported in patients with heart failure, and consequently, their possible role in relation to the severity and mortality of heart failure is unknown.

To analysis the correlation of CTRP3, CTRP9 and HFrEF, we measured levels of CTRP3 and CTRP9 in clinically controlled HFrEF patients of various degrees of severity. Further, in a 3-year follow up, we studied their association with morbidity and mortality.

\section{Methods \\ Study population}

Patients with heart failure with reduced EF that were admitted to the Department of Cardiology at Xijing Hospital, Xian, China, between May 2012 and September 2012, were consecutively recruited for this study. One hundred sixtyeight chronic heart failure patients (122 men and 46 women) were recruited. The diagnosis of HFrEF was made on the basis of clinical history, a chest roentgenogram, an electrocardiogram and an echocardiogram. The inclusion criterion was a diagnosis of heart failure (left ventricular $\mathrm{EF}<40 \%)$. Severity of disease was assessed according to the New York Heart Association (NYHA) standards and fell within functional classes II to IV. Exclusion criteria for the study were hypotension $[\mathrm{SBP} \leq 90 \mathrm{mmHg}(1 \mathrm{mmHg}=$ $0.133 \mathrm{kPa}$ )], cardiogenic shock, severe bradycardia (resting heart rate $\leq 60$ beats $/ \mathrm{min}$ ), atrioventricular block (more than II degree), ongoing acute exacerbation of heart failure, malignancy, acute systemic infections, acute or chronic liver disease or renal failure. The control group paired by age and sex consisted of 176 healthy subjects (116 men and 60 women) who underwent physical examinations, blood analysis and echocardiographic evaluations as a part of a routine health check-up in the health promotion center of Xijing Hospital between May 2012 and June 2012.

\section{Follow up}

The study main end-points were all-cause death and the second outcomes was re-hospitalization rates. Patients were followed up by telephone once every 3 months for a minimum of 36 months. During the whole follow up period, eight patients were lost to follow up. The baseline characteristics of the patients who were lost to follow-up were not significantly different from the others, thus they were not included in the survival analyses.

\section{Laboratory measurements}

After a minimum 8-h overnight fast, venous blood was drawn into EDTA tubes and promptly centrifuged at $4{ }^{\circ} \mathrm{C}$, and plasma was frozen at $-70^{\circ} \mathrm{C}$ for subsequent assays. Plasma glucose, total cholesterol, low-density lipoprotein and high-density lipoprotein cholesterol, triglycerides, serum creatinine, urea nitrogen, uric acid and cystatin $c$ levels were analyzed by applying the standard protocols of the hospital biochemistry laboratory. NT-proBNP was measured by a double-antibody sandwich technique with electrochemiluminescence as signal (Elecsys 2010, Roche Diagnostics). ELISA was used for measurement of CTRP3 and CTRP9 (Shanghai LianShuo Biological Technology Co, China; The intra-assay and interassay coefficients of 
variation were below 5\%). No significant cross-reactivity or interference between human CTRP3 and CTRP9 was observed in our pilot experiment.

\section{Statistical analysis}

Statistical analysis was performed with the Statistical Package for Social Sciences for Windows (IBM, SPSS version 19.0) and graphs were made by Prism 6 (GraphPad software). To observe the possible difference in CTRP3/ CTRP9 levels between healthy control and HFrEF patients, sample size was calculated by PASS 11 (NCSS). Sample sizes of 164 subjects in control group and 164 subjects in HFrEF group achieve $90 \%$ power to detect a difference of -30.0 between the null hypothesis that both group means are 0.0 and the alternative hypothesis that the mean of HFrEF group is 30.0 with estimated group standard deviations of -70.0 and 70.0 and with a significance level (alpha) of 0.01 using a two-sided two-sample t-test. All variables were tested for normality and log transformed was performed for the variables which were not non-normality.

Continuous variables are expressed as mean \pm standard deviation (mean $\pm \mathrm{SD}$ ) unless otherwise stated. Categorical variables were analyzed as value and percentage. Continuous data with a normal distribution were compared with the Student $t$ or ANOVA tests. Chi-square test was used to test for differences in the distribution of categorical variables. Correlations between CTRP3 or CTRP9 levels and other variables were evaluated by Pearson correlation coefficient analysis. Event rates were compared by Kaplan-Meier curves calculated for CTRP3 or CTPR9 above or below the 25th percentile. The independent predictive power of CTRP3 or CTRP9 and other clinical and demographic variables for death and hospitalizations for HFrEF was tested by stepwise Cox proportional hazards regression analyses. The analyses are presented as HR with a 95\% confidence interval (CI). $P$ values $<0.05$ were considered significant.

\section{Results}

\section{Baseline characteristics}

The clinical and biochemical characteristics of the study subjects are presented in Table 1. At total of $168 \mathrm{HFrEF}$ patients were recruited. $65 \%$ of the patients were treated with an ACE inhibitor, $36 \%$ were treated with an angiotensin II antagonist, $69 \%$ received a $\beta$-blocker, $80 \%$ were treated with digoxin and $80 \%$ were using a diuretic. The HFrEF group showed significantly increased BMI, LDL$\mathrm{C}$, triglycerides, serum creatinine and cystatin $\mathrm{c}$ and NTproBNP compared to the control group. LVEF\%, HDL-C and total cholesterol levels in the HFrEF group were

Table 1 Clinical and biochemical characteristics of control and HFrEF groups

\begin{tabular}{|c|c|c|c|}
\hline Variables & Control $(n=176)$ & $\operatorname{HFrEF}(n=168)$ & $P$ \\
\hline Age (years) & $55.11 \pm 13.44$ & $57.25 \pm 14.92$ & 0.418 \\
\hline Sex, male (\%) & $116(65.9 \%)$ & $122(72.6 \%)$ & 0.199 \\
\hline $\mathrm{BMI}\left(\mathrm{Kg} / \mathrm{m}^{2}\right)$ & $21.72 \pm 1.78$ & $23.45 \pm 3.80$ & $<0.001$ \\
\hline Diabetes mellitus (\%) & $0(0 \%)^{a}$ & 40 (23.8\%) & $<0.001$ \\
\hline Hypertension (\%) & $0(0 \%)^{a}$ & $66(39.3 \%)$ & $<0.001$ \\
\hline Myocardial infarction (\%) & $0(0 \%)^{a}$ & $52(31.0 \%)$ & $<0.001$ \\
\hline Systolic pressure $(\mathrm{mmHg})$ & $108.86 \pm 15.70$ & $119.04 \pm 20.71$ & 0.071 \\
\hline Diastolic pressure $(\mathrm{mmHg})$ & $71.41 \pm 10.90$ & $75.96 \pm 12.80$ & 0.577 \\
\hline Fasting glucose (mmol/L) & $4.72 \pm 0.35$ & $5.00 \pm 1.75$ & 0.004 \\
\hline HDL-cholesterol (mmol/L) & $1.31 \pm 0.19$ & $0.93 \pm 0.28$ & $<0.001$ \\
\hline LDL-cholesterol (mmol/L) & $1.67 \pm 0.34$ & $2.33 \pm 0.88$ & $<0.001$ \\
\hline Total Cholesterol (mmol/L) & $4.16 \pm 0.51$ & $3.71 \pm 0.98$ & 0.001 \\
\hline Triglyceride (mmol/L) & $0.95 \pm 0.24$ & $1.39 \pm 0.86$ & $<0.001$ \\
\hline Serum creatinine (umol/L) & $91.11 \pm 6.58$ & $113.79 \pm 32.89$ & $<0.001$ \\
\hline Cystatin C (mg/L) & $1.05 \pm 0.23$ & $1.38 \pm 0.42$ & $<0.001$ \\
\hline NT-proBNP (ng/L) & $3.81 \pm 2.55$ & $9878.69 \pm 9236.14$ & $<0.001$ \\
\hline LVEF (\%) & $59.25 \pm 2.35$ & $28.67 \pm 6.18$ & $<0.001$ \\
\hline CTRP3 (ng/mL) & $236.40 \pm 62.86$ & $173.30 \pm 49.81$ & $<0.001$ \\
\hline CTRP9 (ng/mL) & $180.70 \pm 51.05$ & $124.60 \pm 37.58$ & $<0.001$ \\
\hline
\end{tabular}

Data are expressed as mean \pm SD or frequency (\%)

$P$-values were calculated by an independent-samples t-test or chi-square test

$B M I$ Body mass index, HDL High-density lipoprotein, LDL Low-density lipoprotein, NT-proBNP N-terminal pro-brain natriuretic peptide, LVEF Left ventricular ejection fraction, CTRP3 C1q/TNF-related protein 3, CTRP9 C1q/TNF-related protein 9

${ }^{a}$ These data were acquired by inquiry of medical history 
significantly lower than in the control group. Meanwhile, age, sex, diastolic and systolic blood pressure in these two groups showed no significant differences.

\section{CTRP3 and CTRP9 levels and correlation with clinical parameters}

Compared with control subjects, the concentrations of CTRP3 and CTRP9 were significantly decreased in patients with HFrEF (Table 1, $P<0.001$, respectively). Multivariable linear regression analyses was then preformed. Results analyzed in all enrolled subjects demonstrated that Age, hypertension, NT-ProBNP, LEVF\% were found to be associated with CTRP3 and CTRP9. We than set HFrEF as the outcome variable and CTRP 3/9 and other variables as predictor in a logistic model. Results were shown in Table 2. While HFrEF as the outcome variable, Age, Total Cholesterol, CTRP3 or CTRP9 exhibited statistical significance.

Subsequently, we analyzed relationships between CTRP3/CTRP9 and NYHA class, LVEF\% and NT-proBNP in HFrEF patients. 24.4\% (41 patients) of the patients with HFrEF were NYHA functional class II, $47.0 \%$ (79) were NYHA III and $28.6 \%$ (48) were NYHA IV. We found the more advanced the HFrEF symptom status according to NYHA class, the lower the CTRP3 and CTRP9 concentrations were (Fig. 1a and b). Pearson correlation analysis also revealed that in the HFrEF group, both CTRP3 and CTRP9 concentrations were positively correlated with LVEF\% (CTRP3, $r=0.556, P<0.001$; CTRP9, $r=0.526, P<0.001$ ) and inversely associated with the log index of NT-proBNP levels (CTRP3, $r=-0.454, P<0.001$; CTRP9, $r=-0.483$, $P<0.001$ ) (Fig. 1c to f).

Most human adipose cytokines levels are affected by estrogen. To our surprise, we found that plasma CTRP3 and CTRP9 concentrations were not different according to sex in either the control group or the HFrEF group (Fig. 2a and b). To our surprise, we also observed that CTRP3 and CTRP9 concentrations manifested a significantly positive relationship in both healthy subjects and HFrEF patients (control subjects, $r=0.916, P<0.001$; HFrEF subjects, $r=0.800, P<0.001$ ) (Fig. $2 \mathrm{c}$ and d).

\section{Survival analyses}

To investigate if lower CTRP3/CTRP9 is related with higher death rates or re-hospitalization rates in HFrEF patients, we tried to dichotomize CTRP3/CTRP9 levels for further analysis. Because CTRP3 and CTRP9 are novel adipokines and they have never been reported data showing their concentrations in heart failure patients, we dichotomize CTRP3 and CTRP9 by 25th percentile, 50th percentile and 75th percentile and tested to observe whether there are statistical significance in survive analysis. Results showed that while dichotomize CTRP3 and CTRP9 by 25 th percentile, Kaplan-Meier analysis revealed differences in 3 years of follow-up in death and free-ofhospitalization above and below the first quartile of plasma CTRP3 or CTRP9. Supported by these findings, the CTRP3 and CTRP9 were dichotomize by 25th percentile for further analysis. Clinical and laboratory variables according to 25th percentile of baseline CTRP3 or CTRP9 levels in HFrEF patients are presented in Table 3. We found that patients in the lower quartile of CTRP3 levels manifested lower morbidity related to hypertension, lower rates of LVEF $>25 \%$ and lower NT-proBNP $<20,000 \mathrm{ng} / \mathrm{L}$ and surprisingly younger age. Similarly, patients in the lower CTRP9 quartile were associated with a lower morbidity of hypertension, total cholesterol and lower rate LVEF $>25 \%$ and NT-proBNP $<20,000 \mathrm{ng} / \mathrm{L}$.

During the 3 years of follow-up, 58 (36.3\%) of the patients died in the HFrEF group, whereas 93 (58.1\%) were hospitalized for HFrEF deterioration. Kaplan-Meier analysis revealed differences in event-free events for death and free-of-hospitalization when the group was divided according to concentrations above and below the first quartile of plasma CTRP3 $(136.87 \mathrm{ng} / \mathrm{ml})$ or CTRP9 $(100.75 \mathrm{ng} / \mathrm{ml})$ (Fig. 3). The unadjusted overall mortality risk was markedly elevated in the patients in the first quartile of CTRP3 $(P=0.009)$. This observation was in accordance with the markedly increased HFrEF hospitalization rates in these subjects $(P=0.001)$. Similarly, the unadjusted overall mortality risk and HFrEF hospitalization free rates were also markedly elevated in

Table 2 Logistics regression analyses

\begin{tabular}{|c|c|c|c|c|c|c|c|c|c|}
\hline & \multirow[t]{2}{*}{$P$} & \multirow[t]{2}{*}{ OR } & \multicolumn{2}{|c|}{$95 \% \mathrm{Cl}$. for OR } & & \multirow[t]{2}{*}{$P$} & \multirow[t]{2}{*}{ OR } & \multicolumn{2}{|c|}{$95 \% \mathrm{Cl}$. for OR } \\
\hline & & & Lower & Upper & & & & Lower & Upper \\
\hline Age (years) & 0.000 & 1.121 & 1.064 & 1.182 & Age (years) & 0.000 & 1.134 & 1.068 & 1.205 \\
\hline $\operatorname{Sex}(\%)$ & 0.246 & 1.965 & 0.628 & 6.146 & $\operatorname{Sex}(\%)$ & 0.109 & 2.852 & 0.790 & 10.287 \\
\hline $\mathrm{BMI}\left(\mathrm{kg} / \mathrm{m}^{2}\right)$ & 0.509 & 1.061 & 0.890 & 1.265 & $\mathrm{BMI}\left(\mathrm{kg} / \mathrm{m}^{2}\right)$ & 0.328 & 1.102 & 0.907 & 1.340 \\
\hline Serum Creatinine $(\mu \mathrm{mol} / \mathrm{L})$ & 0.045 & 1.044 & 1.001 & 1.088 & Serum Creatinine $(\mu \mathrm{mol} / \mathrm{L})$ & 0.074 & 1.046 & 0.996 & 1.099 \\
\hline Cystatin C (mg/L) & 0.387 & 0.504 & 0.107 & 2.379 & Cystatin C (mg/L) & 0.642 & 0.680 & 0.134 & 3.458 \\
\hline Total cholesterol (mmol/L) & 0.000 & 0.251 & 0.117 & 0.540 & Total cholesterol (mmol/L) & 0.001 & 0.244 & 0.103 & 0.581 \\
\hline Triglyceride (mmol/L) & 0.002 & 23.336 & 3.264 & 166.867 & Triglyceride $(\mathrm{mmol} / \mathrm{L})$ & 0.001 & 48.693 & 4.998 & 474.405 \\
\hline CTRP3 (ng/mL) & 0.000 & 0.977 & 0.967 & 0.987 & CTRP9 (ng/mL) & 0.000 & 0.960 & 0.945 & 0.977 \\
\hline
\end{tabular}



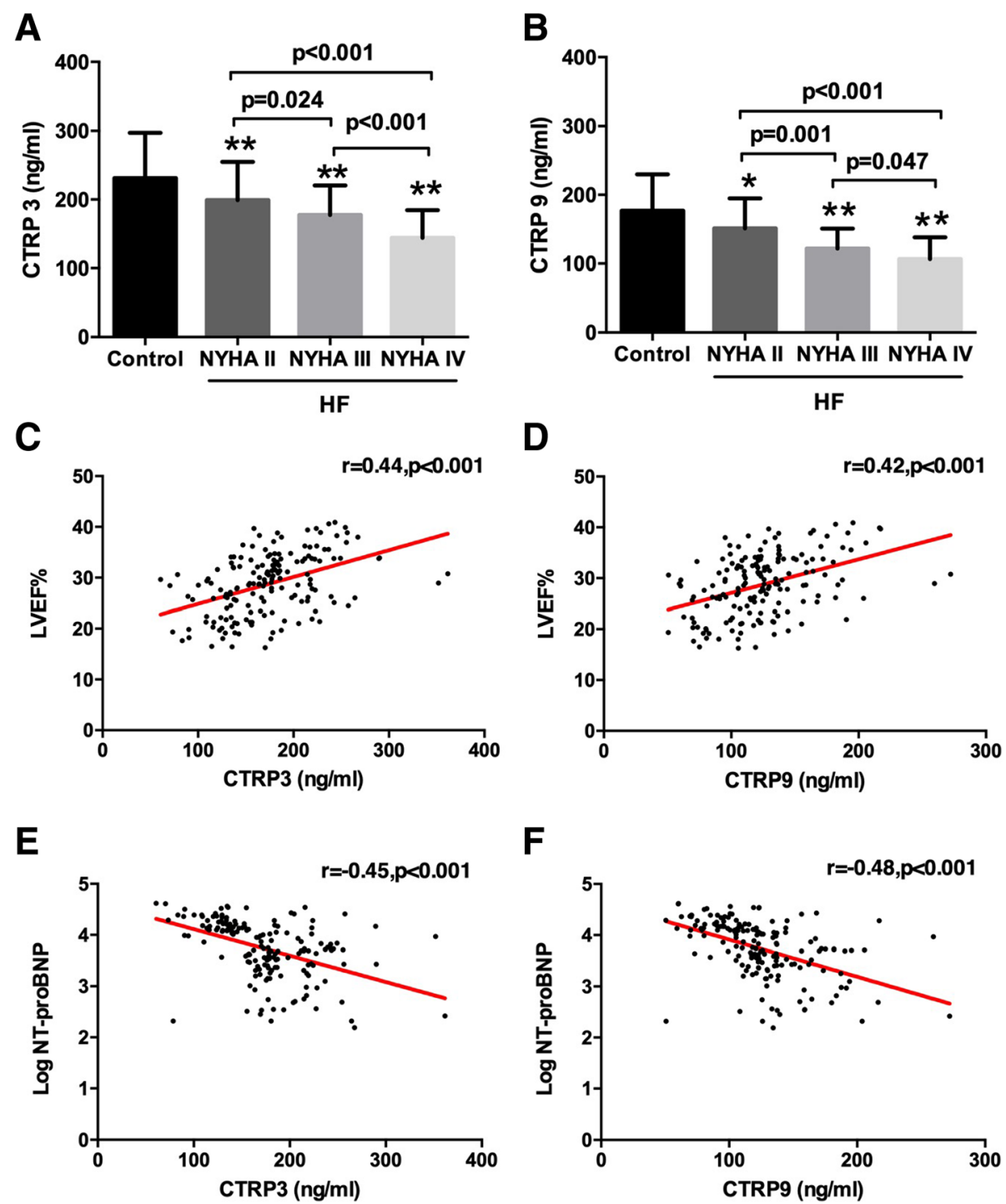

$\mathbf{F}$

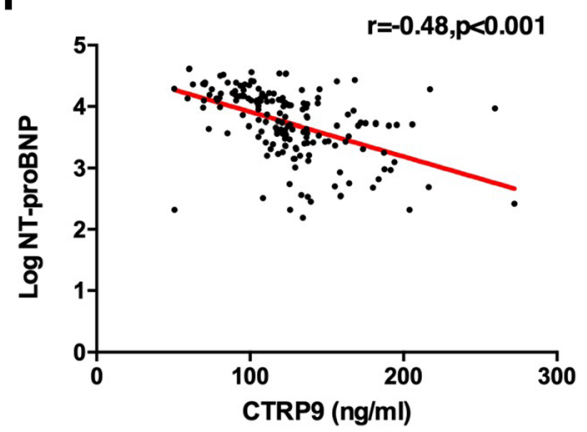

Fig. 1 Concentration of CTRP3 (a) and CTRP9 (b) according to the severity of HFrEF stratified by NYHA functional class. Scatterplots of the association between CTRP3/CTRP9 and LVEF\% (c and $\mathbf{d}$ ) and with log transformed values of NT-proBNP (e and $\mathbf{f}$ ) in HFrEF patients. ${ }^{*} p<0.05$, ${ }^{* *} p<0.01$ vs. Control group

the subjects in the first quartile of CTRP9 $(P<0.001$, respectively).

Multivariate analysis by stepwise Cox proportional hazards regression analysis confirmed that plasma CTRP3 concentrations (determined dichotomously, adjusted for age, hypertension, $\quad$ LVEF $>25 \%, \quad$ NT-proBNP $<20,000 \mathrm{ng} / \mathrm{L}$ ) remained independently predictive of death with a hazard risk ratio of 1.93 (95\% CI 1.03 to $3.62, P=0.042$ ). Moreover, CTPR3 concentration was also an independent predictor of HFrEF hospitalization free rates during the follow up period with a hazard risk ratio of 2.34 ( $95 \%$ CI 1.43 to $3.82 P=$ 0.001). Similarly, CTRP9 (determined dichotomously, adjusted for hypertension, diabetes, LVEF $>25 \%$, NT-proBNP< $20,000 \mathrm{ng} / \mathrm{L}$, diastolic pressure and total cholesterol) was also found to be independently predictive of death with a hazard risk ratio of 1.98 ( $95 \% \mathrm{CI} 1.02$ to $3.85, P=0.044)$ and a predictor of free of HFrEF hospitalization with a hazard risk ratio of 2.67 ( $95 \%$ CI 1.58 to $4.50, P<0.001$ ).

\section{Discussion}

Heart failure is emerging rapidly as a major cause of morbidity and mortality around the world. As such, novel means of stratifying disease severity are needed. Here, in a prospective cohort of patients with HFrEF, we report that CTRP3 and CTRP9 levels are decreased in patients with HFrEF in a manner proportionate to symptom severity (NYHA class). Consistent with that, levels of these 2 adipokines were inversely associated with markers of HFrEF severity, such as LVEF and NT-proBNP. We also observed a significant relationship between CTRP3 and CTRP9 

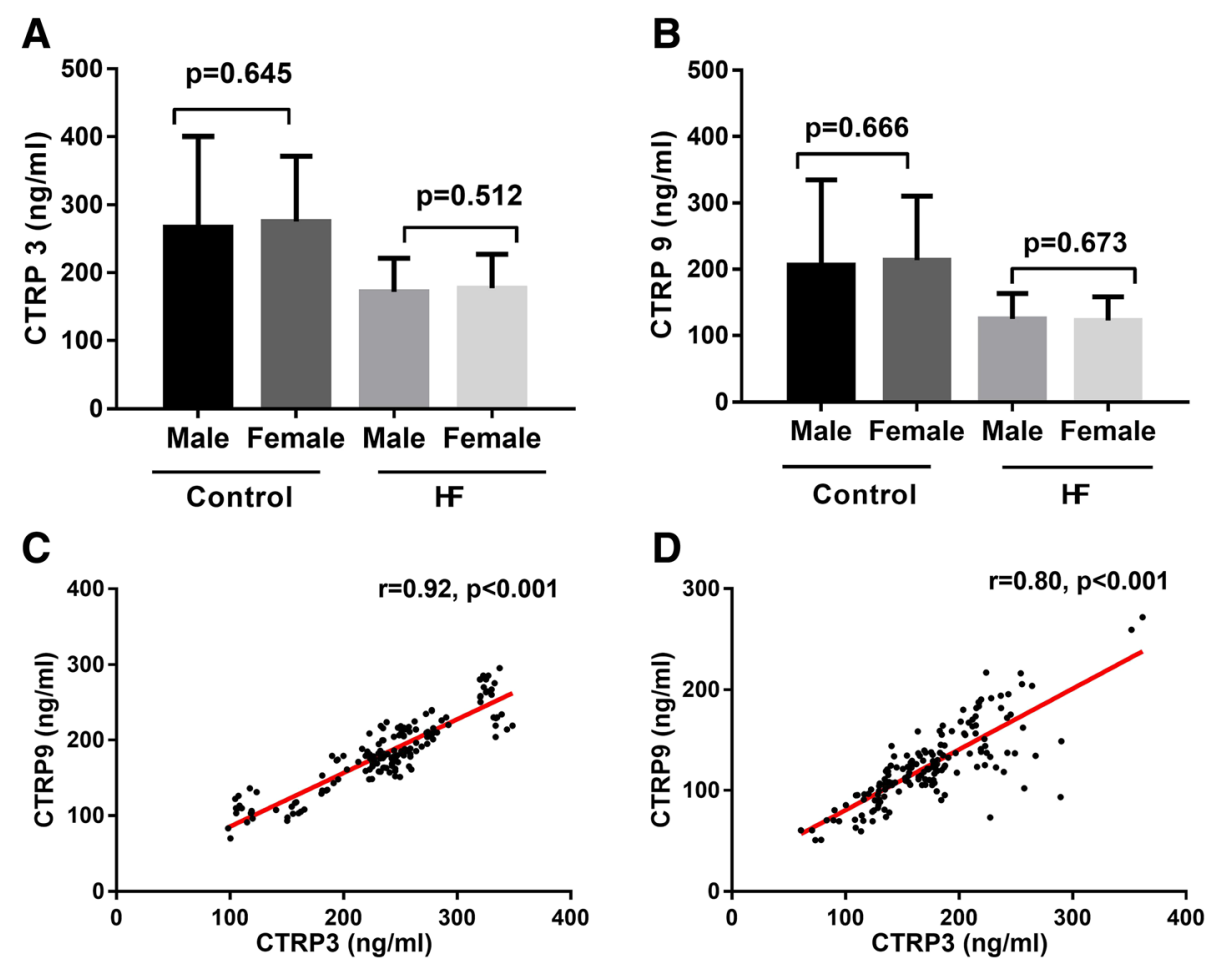

Fig. 2 Distribution of CTRP3 and CTRP9 according to sex (a and b). Correlation between CTRP3 and CTRP9 in control group (c) and HFrEF group (d)

Table 3 Baseline characteristics according to 25 percentile of CTRP3 and CTRP9 levels

\begin{tabular}{|c|c|c|c|c|c|c|}
\hline \multirow[t]{2}{*}{ Variables } & \multicolumn{3}{|l|}{ CTRP3 (ng/ml) } & \multicolumn{3}{|l|}{ CTRP9 (ng/ml) } \\
\hline & $\leq 136.87(n=42)$ & $>136.87(n=126)$ & $P$ & $\leq 100.75(n=42)$ & $>100.75(n=126)$ & $P$ \\
\hline Age (years) & $51.74 \pm 19.16$ & $59.09 \pm 12.80$ & 0.019 & $51.48 \pm 17.58$ & $59.17 \pm 13.47$ & 0.142 \\
\hline Sex,male (\%) & $32(76.2 \%)$ & $90(71.4 \%)$ & 0.549 & $31(73.8 \%)$ & $91(72.2 \%)$ & 0.842 \\
\hline BMI $\left(\mathrm{kg} / \mathrm{m}^{2}\right)$ & $22.80 \pm 3.86$ & $23.66 \pm 3.77$ & 0.933 & $22.30 \pm 3.59$ & $23.83 \pm 3.80$ & 0.462 \\
\hline Diabetes mellitus (\%) & $7(16.7 \%)$ & $33(26.2 \%)$ & 0.209 & $6(14.3 \%)$ & $34(27.0 \%)$ & 0.094 \\
\hline Hypertension (\%) & $10(23.8 \%)$ & $56(44.4 \%)$ & 0.018 & $7(16.7 \%)$ & $59(46.8 \%)$ & 0.001 \\
\hline Myocardial infarction (\%) & $12(28.6 \%)$ & $40(31.7)$ & 0.700 & $9(21.4 \%)$ & $43(34.1 \%)$ & 0.123 \\
\hline Disease duration (months) & $44.40 \pm 56.92$ & $33.21 \pm 36.44$ & 0.237 & $38.81 \pm 42.59$ & $35.07 \pm 42.70$ & 0.574 \\
\hline Systolic pressure $(\mathrm{mmHg})$ & $113.05 \pm 22.334$ & $121.03 \pm 19.83$ & 0.287 & $111.71 \pm 20.92$ & $121.48 \pm 20.14$ & 0.822 \\
\hline Diastolic pressure $(\mathrm{mmHg})$ & $73.45 \pm 14.61$ & $76.80 \pm 12.09$ & 0.443 & $71.43 \pm 9.98$ & $77.48 \pm 13.30$ & 0.021 \\
\hline Fasting glucose (mmol/L) & $4.75 \pm 1.43$ & $5.08 \pm 1.83$ & 0.275 & $4.45 \pm 1.10$ & $5.18 \pm 1.88$ & 0.059 \\
\hline HDL-cholesterol (mmol/L) & $0.86 \pm 0.26$ & $0.96 \pm 0.29$ & 0.865 & $0.87 \pm 0.30$ & $0.95 \pm 0.28$ & 0.486 \\
\hline LDL-cholesterol (mmol/L) & $2.11 \pm 0.84$ & $2.40 \pm 0.88$ & 0.839 & $2.12 \pm 0.83$ & $2.40 \pm 0.89$ & 0.731 \\
\hline Total Cholesterol (mmol/L) & $3.45 \pm 1.00$ & $3.80 \pm 0.96$ & 0.842 & $3.45 \pm 0.98$ & $3.80 \pm 0.97$ & 0.015 \\
\hline Triglyceride (mmol/L) & $1.27 \pm 0.71$ & $1.42 \pm 0.90$ & 0.332 & $1.15 \pm 0.56$ & $1.47 \pm 0.92$ & 0.873 \\
\hline Serum creatinine (umol/L) & $123.69 \pm 36.954$ & $110.49 \pm 30.89$ & 0.214 & $119.33 \pm 34.45$ & $111.94 \pm 32.30$ & 0.547 \\
\hline Cystatin c (mg/L) & $1.48 \pm 0.43$ & $1.35 \pm 0.42$ & 0.428 & $1.40 \pm 0.39$ & $1.37 \pm 0.44$ & 0.828 \\
\hline NT-proBNP<20,000 (ng/L) & $25(59.5 \%)$ & $121(96 \%)$ & $<0.001$ & $27(64.3 \%)$ & 119(94.4\%) & $<0.001$ \\
\hline LVEF> 25\% & 19 (45.2\%) & 103(81.7\%) & $<0.001$ & $21(50 \%)$ & $101(80.2 \%)$ & $<0.001$ \\
\hline
\end{tabular}


A

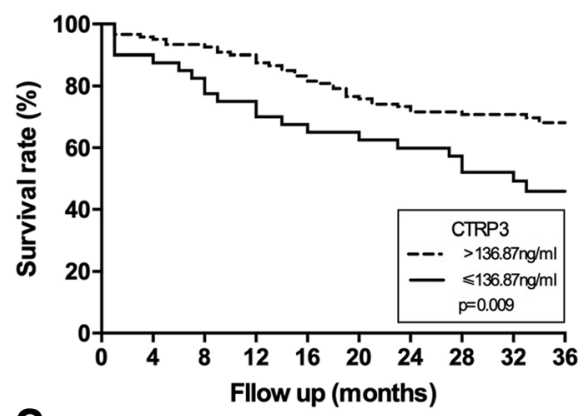

C

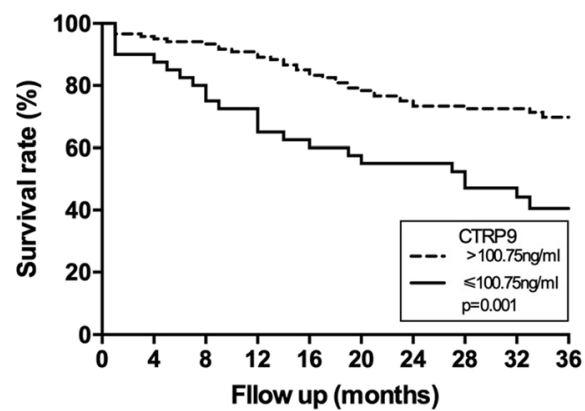

B

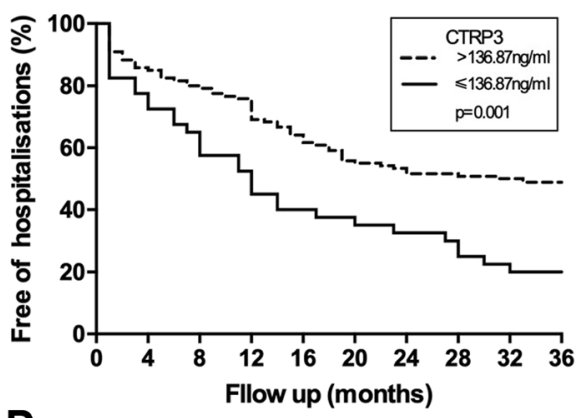

D

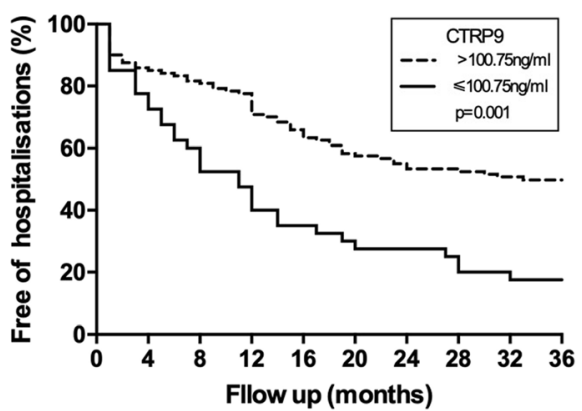

Fig. 3 Kaplan-Meier survival curves according to the above or below 25th centile of CTRP3 or CTRP9 concentrations. a Event free survival rate of CTRP3. b Free of HFrEF hospitalization rates of CTRP3. c Event free survival rate of CTRP9. d Free of HFrEF hospitalization rates of CTRP9

levels. Finally, decreased levels of CTRP3 and CTRP9 were each associated with increased mortality and hospitalization.

CTRP is a widely expressed and highly conserved adipokine family of adiponectin paralogs [18]. The number of proteins in the CTRP family is rapidly growing and currently comprises 15 members in addition to adiponectin [6]. CTRPs principally form highly stable, biologically active homotrimers and control adipocyte physiology as well as energy homeostasis [18]. In previous preclinical research, using an in vivo mouse myocardial ischemia model, we found that supplementing CTRP3 [7] or CTRP9 [9] can attenuate post-MI remodeling and augment post-MI contractile function through their anti-apoptotic and pro-angiogenic properties. However, little is known about the levels of CTRP3 and CTRP9 in patients with disease. In previous studies, data suggested that patients with acute coronary syndrome or stable angina pectoris had significantly lower plasma CTRP3 concentrations compared with control subjects [8]. In addition, previous data also showed decreased CTRP3 in obese populations [19] . No data have existed regarding CTRP9 in patients with cardiovascular disorders, but there are statistics showing that CTRP9 concentrations are decreased in patients with metabolic syndrome [20], but surprisingly elevated in obesity [21]. Although still under debate, some researches indicated that CTRP3 also share a similar pattern. CTRP3 might increase in obese subjects but decrease in T2DM patients [22-24].
In the present study, we observed that low CTRP3 or CTRP9 levels are associated with a higher likelihood of death and morbidity. Taken together with our data in previous basic research, CTRP3/CTRP9 may be a potential therapeutic and diagnostic target.

CTRP3 and CTRP9 share a similar modular organization with adiponectin [18], which is an insulin-sensitizing adipokine with anti-inflammatory and anti-atherogenic properties [25]. In the present study, we found CTRP3 and CTRP9 concentrations to be decreased in HFrEF patients, with the concentration negatively related to NYHA class. However, evidence exists that APN levels are increased in HFrEF patients [4]. In two previous cross-sectional studies, researchers found in healthy subjects, CTRP3 $(r=0.194$, $p<0.001)$ and CTRP9 $(r=0.15, p<0.03)$ are positively correlated with adiponectin, respectively. Therefore, we presume that although CTRP3, CTRP9 and APN have similar biological activities and CTRP3/CTRP9 concentration are positively related to the APN in healthy subjects, their relationship is not a causal relationship and the signaling pathways regulating the level of APN and CTRP3 and CTPR9 may be different.

Some research reported that the mRNA of CTRP3 in fat tissue, and its serum levels are similar in both sex in mice [26]. However, clinical trials showed that CTRP3 concentration was significantly higher in women than in men [27]. Similarly, an animal study examining the level of CTRP9 showed that female mice had higher levels 
than male mice [15]. Interestingly, contrary to the results of the animal study, in a human study, CTRP9 concentration was not different according to sex [20]. We found that in both healthy people or in patients with HFrEF, the concentration of CTRP3 and CTRP9 were not different in male and female. Our data support that CTRP3 and CTRP9 levels are similar in both sexes.

Each member in the CTRP family has its own unique biological activity [28]. However, to our knowledge, the relationships among CTPRs in the CTRP family have never been reported. The present study is the first to demonstrate that CTRP3 and CTRP9 levels have a significantly positive relationship in both healthy subjects and HFrEF patients. Previous data suggest that CTRP3 reduces tumor necrosis factor- $\alpha$ (TNF- $\alpha$ ) and interleukin-6 (IL-6) secretion through suppression of nuclear factor $k B$ signaling pathway [29]. Moreover, TNF $\alpha$ can inhibit CTRP9 expression via oxidative stress-mediated inhibition of transcription factor PPAR $\gamma$ [30]. Thus, TNF- $\alpha$ might be the link in regulating their levels in adipose tissue. In addition, we have reported that supplementing either CTRP3 or CTRP9 can attenuate post-MI remodeling and augment post-MI contractile function. In these experiments, whether replenishing one has an impact on the other during the MI process to exert an anti-apoptotic or pro-angiogenic effect remains unknown.

Several limitations exist in this study. First, this study enrolled a relatively small cohort of subjects from a single center. All were of Chinese ancestry. As such, generalizability to other groups of patients must be evaluated going forward. That said, our prospective study design in which we enrolled consecutive patients mitigates some of these limitations. Also, we did not measure changes in CTRP3/CTRP9 nor clinical and biochemical characteristics during follow-up, and hence, no causality of the interrelationship between these parameters can be determined. Thirdly, because this is the first study to examine CTRP3 and CTRP9 in relation to prognosis in $\mathrm{HFrEF}$, the present findings should be confirmed in other studies.

\section{Conclusion}

We demonstrate that CTRP3 and CTRP9 are each decreased in patients with HFrEF, proportionate to disease severity, and each is associated with increased morbidity and mortality.

\section{Abbreviations}

BNP: Brain natriuretic peptide; CHF: Chronic heart failure; CTRP3: C1q/TNFrelated protein 3; CTRP9: C1q/TNF-related protein 9; HFrEF: Heart failure with reduced ejection fraction; NT-proBNP: N-terminal pronatriuretic peptide

\section{Acknowledgments}

The authors thank the patients enrolled in this study for their participation in data collection and assessment. The authors also wish to thank Dr. Stephen Ellis from Cleveland Clinic, US, Dr. Joseph Hill from UT Southwestern Medical Center, US and Prof. Jielai Xia from FMMU, China for editing this manuscript.

\section{Authors' contributions}

GC and ZS performed the statistical analyses, evaluated the results and drafted the paper. LK, WS, MX, FF and TL participated in the conception and design of the study. WR, SR contributed to the enrollment of the patients and follow up. MB and TZ contributed to analyzing data. All authors have read and approved the final manuscript.

\section{Funding}

This work was financially supported by the National key R \& D plan (Grant No. 2018YFA0107400), the Program for National Science Funds of China (Grants No. 81600281, 81600310, 81300078), Program for Changjiang Scholars and Innovative Research Teamin University (Grant No. PCSIRT-14R08), National Natural Science Foundation of China-Youth Projects (No.81500272) and Science and Technology Development of Research Project of Shaanxi Province-Key Problems of Science and Technology in Social Development (No.2016SF-034). The funding were used for the reagent, patients enrollment, academic communication, labor costs and so on.

\section{Availability of data and materials}

The datasets used and/or analyzed during the current study available from the corresponding author on reasonable request.

\section{Ethics approval and consent to participate}

The study adhere to CONSORT guidelines. The local ethics committee of Xijing Hospital approved the study and registered online (ClinicalTrials.gov: NCT01372800). The investigation conforms with the principles outlined in the Declaration of Helsinki and written informed consent was obtained from all participants.

\section{Consent for publication}

Not applicable.

\section{Competing interests}

The authors declare that they have no competing interests.

\section{Author details}

${ }^{1}$ Department of Cardiology, Xijing Hospital, The Fourth Military Medical University, 15 Changle West Road, Xi'an 710032, China. ${ }^{2}$ Department of Epidemiology and Biostatistic, School of Public Health, Xi'an Jiaotong University Health Science Center, No.76, Yanta West Road, Xi'an 710061, China. ${ }^{3}$ Department of Statistics, The Fourth Military Medical University, 15 Changle West Road, Xi'an 710032, China. ${ }^{4}$ Department of Physiology and Pathophysiology, The Fourth Military Medical University, 15 Changle West Road, Xi'an 710032, China. ${ }^{5}$ Center for Translational Medicine, Thomas Jefferson University, Philadelphia, PA 19107, USA.

Received: 16 August 2018 Accepted: 22 May 2019

Published online: 10 June 2019

\section{References}

1. Levy D, Kenchaiah S, Larson MG, Benjamin EJ, Kupka MJ, Ho KK, et al. Longterm trends in the incidence of and survival with heart failure. N Engl J Med. 2002;347(18):1397-402.

2. Roger VL, Weston SA, Redfield MM, Hellermann-Homan JP, Killian J, Yawn $\mathrm{BP}$, et al. Trends in heart failure incidence and survival in a communitybased population. JAMA. 2004;292(3):344-50.

3. Friedman JM, Halaas JL. Leptin and the regulation of body weight in mammals. Nature. 1998;395(6704):763-70.

4. George J, Patal S, Wexler D, Sharabi Y, Peleg E, Kamari Y, et al. Circulating adiponectin concentrations in patients with congestive heart failure. Heart. 2006;92(10):1420-4.

5. Ryo M, Nakamura T, Kihara S, Kumada M, Shibazaki S, Takahashi M, et al. Adiponectin as a biomarker of the metabolic syndrome. Circ J. 2004;68(11): 975-81.

6. Schaffler A, Buechler C. CTRP family: linking immunity to metabolism. Trends Endocrinol Metab. 2012;23(4):194-204.

7. Yi W, Sun Y, Yuan Y, Lau WB, Zheng Q, Wang $X$, et al. C1q/tumor necrosis factor-related protein-3, a newly identified adipokine, is a novel antiapoptotic, proangiogenic, and cardioprotective molecule in the ischemic mouse heart. Circulation. 2012;125(25):3159-69. 
8. Choi KM, Hwang SY, Hong HC, Choi HY, Yoo HJ, Youn BS, et al. Implications of C19/TNF-related protein-3 (CTRP-3) and progranulin in patients with acute coronary syndrome and stable angina pectoris. Cardiovasc Diabetol. 2014;13:14.

9. Sun Y, Yi W, Yuan Y, Lau WB, Yi D, Wang X, et al. C1q/tumor necrosis factorrelated protein-9, a novel adipocyte-derived cytokine, attenuates adverse remodeling in the ischemic mouse heart via protein kinase a activation. Circulation. 2013;128(11 Suppl 1):S113-20.

10. Schmid A, Kopp A, Hanses F, Bala M, Muller M, Schaffler A. The novel adipokine C1q/TNF-related protein-3 is expressed in human adipocytes and regulated by metabolic and infection-related parameters. Exp Clin Endocrinol Diabetes. 2012;120(10):611-7.

11. Peterson JM, Wei Z, Wong GW. C1 / /TNF-related protein-3 (CTRP3), a novel adipokine that regulates hepatic glucose output. J Biol Chem. 2010;285(51): 39691-701.

12. Compton SA, Cheatham B. CTRP-3: blocking a toll booth to obesity-related inflammation. Endocrinology. 2010;151(11):5095-7.

13. Kopp A, Bala M, Buechler C, Falk W, Gross P, Neumeier M, et al. C1q/TNFrelated protein-3 represents a novel and endogenous lipopolysaccharide antagonist of the adipose tissue. Endocrinology. 2010;151(11):5267-78

14. Maeda T, Wakisaka S. CTRP3/cartducin is induced by transforming growth factor-beta1 and promotes vascular smooth muscle cell proliferation. Cell Biol Int. 2010;34(3):261-6.

15. Wong GW, Krawczyk SA, Kitidis-Mitrokostas C, Ge G, Spooner E, Hug C, et al. Identification and characterization of CTRP9, a novel secreted glycoprotein, from adipose tissue that reduces serum glucose in mice and forms heterotrimers with adiponectin. FASEB J. 2009;23(1):241-58.

16. Zheng $Q$, Yuan $Y, Y i$ W, Lau WB, Wang Y, Wang $X$, et al. C1q/TNF-related proteins, a family of novel adipokines, induce vascular relaxation through the adiponectin receptor-1/AMPK/eNOS/nitric oxide signaling pathway. Arterioscler Thromb Vasc Biol. 2011;31(11):2616-23.

17. Kambara T, Ohashi K, Shibata R, Ogura Y, Maruyama S, Enomoto T, et al. CTRP9 protein protects against myocardial injury following ischemiareperfusion through AMP-activated protein kinase (AMPK)-dependent mechanism. J Biol Chem. 2012;287(23):18965-73.

18. Wong GW, Wang J, Hug C, Tsao TS, Lodish HF. A family of Acrp30/ adiponectin structural and functional paralogs. Proc Natl Acad Sci U S A. 2004;101(28):10302-7.

19. Wolf RM, Steele KE, Peterson LA, Magnuson TH, Schweitzer MA, Wong GW. Lower circulating C1q/TNF-related Protein-3 (CTRP3) levels are associated with obesity: a cross-sectional study. PLoS One. 2015;10(7):e0133955.

20. Hwang YC, Woo Oh S, Park SW, Park CY. Association of serum C1q/TNFrelated Protein-9 (CTRP9) concentration with visceral adiposity and metabolic syndrome in humans. Int J Obes. 2014;38(9):1207-12.

21. Wolf RM, Steele KE, Peterson LA, Zeng X, Jaffe AE, Schweitzer MA, et al. C1q/ TNF-related Protein-9 (CTRP9) levels are associated with obesity and decrease following weight loss surgery. J Clin Endocrinol Metab. 2016;101(5):2211-7.

22. Fadaei R, Moradi N, Baratchian M, Aghajani H, Malek M, Fazaeli AA, et al. Association of C19/TNF-related Protein-3 (CTRP3) and CTRP13 serum levels with coronary artery disease in subjects with and without type 2 diabetes mellitus. PLoS One. 2016;11(12):e0168773.

23. Wagner RM, Sivagnanam K, Clark WA, Peterson JM. Divergent relationship of circulating CTRP3 levels between obesity and gender: a cross-sectional study. PeerJ. 2016;4:e2573.

24. Deng W, Li C, Zhang Y, Zhao J, Yang M, Tian M, et al. Serum C1q/TNFrelated protein-3 (CTRP3) levels are decreased in obesity and hypertension and are negatively correlated with parameters of insulin resistance. Diabetol Metab Syndr. 2015;7:33.

25. Turer AT, Scherer PE. Adiponectin: mechanistic insights and clinical implications. Diabetologia. 2012;55(9):2319-26.

26. Wong GW, Krawczyk SA, Kitidis-Mitrokostas C, Revett T, Gimeno R, Lodish HF. Molecular, biochemical and functional characterizations of $\mathrm{Clq} / \mathrm{TNF}$ family members: adipose-tissue-selective expression patterns, regulation by PPAR-gamma agonist, cysteine-mediated oligomerizations, combinatorial associations and metabolic functions. Biochem J. 2008:416(2):161-77.

27. Choi HY, Park JW, Lee N, Hwang SY, Cho GJ, Hong HC, et al. Effects of a combined aerobic and resistance exercise program on C1q/TNF-related protein-3 (CTRP-3) and CTRP-5 levels. Diabetes Care. 2013;36(10):3321-7.

28. Seldin MM, Tan SY, Wong GW. Metabolic function of the CTRP family of hormones. Rev Endocr Metab Disord. 2014;15(2):111-23.
29. Weigert J, Neumeier M, Schaffler A, Fleck M, Scholmerich J, Schutz C, et al. The adiponectin paralog CORS-26 has anti-inflammatory properties and is produced by human monocytic cells. FEBS Lett. 2005;579(25):5565-70.

30. Su H, Yuan $Y$, Wang $X M$, Lau WB, Wang $Y$, Wang $X$, et al. Inhibition of CTRP9, a novel and cardiac-abundantly expressed cell survival molecule, by TNFalpha-initiated oxidative signaling contributes to exacerbated cardiac injury in diabetic mice. Basic Res Cardiol. 2013;108(1):315.

\section{Publisher's Note}

Springer Nature remains neutral with regard to jurisdictional claims in published maps and institutional affiliations.
Ready to submit your research? Choose BMC and benefit from:

- fast, convenient online submission

- thorough peer review by experienced researchers in your field

- rapid publication on acceptance

- support for research data, including large and complex data types

- gold Open Access which fosters wider collaboration and increased citations

- maximum visibility for your research: over $100 \mathrm{M}$ website views per year

At BMC, research is always in progress.

Learn more biomedcentral.com/submissions 